УДК 551.509.39

DOI: https://doi.org/

ISSN 1995-5499

Поступила в редакцию 20.10.2020

Подписана в печать 02.02.2021

\title{
ИДЕНТИФИКАЦИЯ КОРРЕЛЯЦИОННЫХ СВЯЗИЕЙ МЕЖДУ МЕТЕОРОЛОГИЧЕСКИМИ ПАРАМЕТРАМИ И СЕЙСМИЧЕСКИМ ШУМОМ
}

\author{
() 2020 М. Е. Семенов ${ }^{1,3}$, М. Б. Мозиков ${ }^{\bowtie 2}$, И. Н. Сафронич ${ }^{1,4}$ \\ ${ }^{1}$ Военный учебно-научный центр Военно-воздушных сил \\ «Военно-воздушная академия им. проф. Н. Е. Жуковского и Ю. А. Гагарина» \\ ул. Старых Большевиков, 54 a, 394064 Воронеж, Российская Федерация \\ ${ }^{2}$ Главный гидрометеорологический центр Министерства обороны Российской Федераиии \\ Фрунзенская наб., 22/2, 119146 Москва, Российская Федераиия \\ ${ }^{3}$ Воронежский государственный университет \\ Университетская пл., 1, 394018 Воронеж, Российская Федерация \\ ${ }^{4}$ Единая геобизическая служба Российской академии наук \\ пр-т Ленина, 189, 249035 Обнинск, Российская Федерация
}

\begin{abstract}
Аннотация. В работе представлен анализ корреляционных связей между микросейсмическим фоном и вариациями атмосферного давления на территории Воронежского кристаллического массива, которая является платформенным регионом. Цель данного анализа - разработка теоретической основы для методики прогноза погоды в условиях ограниченной метеорологической информации на основе регистрации колебаний отфильтрованного микросейсмического шума - микросейсмического фона.

Однако фильтрация помех, присутствующих в микросейсмическом шуме представляет собой нетривиальную задачу. Основной проблемой изучения фонового микросейсмического поля на платформенной территории является высокий уровень помех, превышающий в несколько раз амплитуды фоновых колебаний. Это не позволяет стандартными методами полностью исключить влияние помех на полезный сигнал, а использование для расчёта «хороших» интервалов делает получаемый результат субъективным, снижая его достоверность, что ограничивает применимость фонового микросейсмического поля в качестве инструментария для изучения платформенных территорий. В данной работе обработка данных произведена с использованием метода осредненного спектра и метода минимальных амплитуд. Также, в целях снижения влияния антропогенной нагрузки на микросейсмический шум, были отобраны колебания, зарегистрированные в ночной период времени.

В ходе исследования выполнен сравнительный анализ используемых методов, выявлен наиболее оптимальный подход для проведения дальнейших исследований при анализе взаимодействия микросейсмического фона и вариаций метеорологических характеристик. Им является метод минимальных амплитуд. Выявлено наличие умеренной и заметной (по шкале Чеддока) корреляционной связи между рассматриваемыми параметрами. Также отмечено различие в тесноте этой связи между станциями, принадлежащими одному кристаллическому массиву. Как правило, наиболее выраженная связь между микросейсмическим фоном и атмосферным давлением наблюдалась в диапазоне до 3 Гц и в диапазоне от 4-6 до 18-20 Гц.
\end{abstract}

Мозиков Михаил Борисович e-mail: mb.mozikov@gmail.com

Ключевые слова: микросейсмический фон, микросейсмы, атмосферное давление, корреляционные связи.

(i) Контент доступен под лицензией Creative Commons Attribution 4.0 License. The content is available under Creative Commons Attribution 4.0 License. 
Идентификация корреляционньх связей между метеорологическими параметрами и сейсмическим...

\section{ВВЕДЕНИЕ}

В последнее время проблеме изучения микросейсмического поля и его реализации, микросейсмическому фону, уделяется повышенное внимание исследователей [1-5]. Микросейсмическое поле в пункте наблюдения создает сложный волновой процесс - микросейсмический шум, который является суперпозицией колебаний значительного числа природных и антропогенных источников.

В совместной лаборатории ФИЦ ЕГС РАН и ВГУ, проводившей изучение микросейсмического поля Воронежского кристаллического массива (ВКМ) на основе анализа трёхкомпонентных записей микросейсмического шума установлено, чтокроме антропогенной нагрузки, «сейсмических бурь», волновых полей от взрывов и землетрясений на записях присутствует процесс - микросейсмический фон (МФ), который отражает вариации фонового микросейсмического поля (ФМП). В качестве оцениваемого параметра использовался уровень микросейсмического шума - средняя амплитуда на выбранном интервале и в заданном диапазоне частот [6].

Основной проблемой изучения ФМП на платформенной территории является высокий уровень помех, превышающий в несколько раз амплитуды фоновых колебаний. Это не позволяет стандартными методами полностью исключить влияние помех на полезный сигнал, а использование для расчёта «хороших» интервалов делает получаемый результат субъективным, снижая его достоверность, что ограничивает применимость фонового микросейсмического поля в качестве инструментария для изучения платформенных территорий.

Исследование микросейсмического фона в пунктах наблюдения на ВКМ показало, что в отличие от других процессов присутствующих в микросейсмическом шуме только вариация его уровня является непрерывным, широкополосным и однородным процессом [6]. Это значит что, только МФ на записях присутствует всегда, отсутствуют диапазоны частот, в которых не было бы его составляющих, а рассчитанная для уровня МФ средняя амплитуда каждой гармоники является величиной, т. е. он является «постоянной» составляющей в микросейсмическом шуме.

Несмотря на информативность рассматриваемого процесса, физическая природа микросейсмического фона, так же, как и связь с другими геофизическими параметрами, остается дискуссионным вопросом.

По поводу механизма генерации микросейсм существует несколько точек зрения [78]. В частности, высказаны идеи об образовании микросейсм в результате удара морских волн о крутые берега, о возбуждении микросейсм в результате передачи энергии морских волн на дно океана в центре циклона, в какой бы части океана он ни находился, а так же об образовании микросейсм стоячими волнами на поверхности водных бассейнов. Области генерации микросейсм в соответствии с механизмом их образования приурочены к прибрежным зонам. Однако, морские волны не определяют полностью периодов микросейсм, а являются лишь одним из многих факторов, влияющих на спектр микросейсм. Отношение периодов морских волн к периодам микросейсм варьируют в весьма широких пределах. Форма спектра микросейсм определяется главным образом строением земной коры в некоторой окрестности точки наблюдения микросейсмических волн.

Изучение условий распространения микросейсм позволило выявить их слабое затухание при распространении в континентальной земной коре, что имеет большое значение в рамках проводимого исследования. Кроме того, было установлено, что в микросейсмическом фоне находят свое отражениевариации атмосферного давления, обусловленные эволюцией и перемещением крупных барических образований над океанами, а также прохождением барических образований и атмосферных фронтов непосредственно над геофизической станцией [5].

В рамках настоящей работы изучается связь вариаций амплитуды микросейсмического фона и атмосферного давления в пространственно сопряженных точках наблюдения.

Одной из традиционных задач метеорологии является совершенствование методов 
прогнозирования в условиях ограниченной информации. Но существующие на данный момент методикиоснованы преимущественно на сопоставлении синоптической ситуации на территории, с которой поступают данные приземных и аэрологических наблюдений, с классическими синоптическими ситуациями. Описанный подход имеет ряд существенных недостатков. Во-первых, классические синоптические ситуации не подвергались уточнению на протяжении большого периода времени, тогда как глобальные и локальные изменения климата требуют более частого повторного анализа. Во-вторых, изменилась граница и размеры территории, потенциально доступной для проведения наблюдений и измерений в границах территории Российской Федерации. В третьих, предложенные методы требуют чрезвычайно высокой квалификации специалиста-синоптика, разрабатывающего прогноз.

Поэтому, решение проблемы прогноза погоды в условиях ограниченной информации требует разработки новых методических подходов. В свою очередь, вариации микросейсмического фона, связанные с прохождением барических образований могут стать важным источником информации (предиктором?).

В работе [5] проанализировано влияние барических вариаций разного происхождения на характеристики сейсмического фона в диапазоне частот от 0,03 до 20 Гц. Выявлено наличие корреляционной связи сейсмического фона как с вариациями атмосферного давления, связанными с циклонической деятельностью, вызывающей сильную штормовую активность в Атлантике, так и с вариациями, связанными непосредственно с прохождением циклона или атмосферного фронта над сейсмической станцией MHV сети станций IRIS Global Seismic Network. Коэффициент линейной корреляционной связи временных вариаций амплитуды микросейсмических колебаний и атмосферного давления составил $K=0,65$ при значимости $r=0,95$.

Очевидно, что рассматриваемые связи на других станциях станций IRIS Global Seismic Network могут быть отличны Цель данной работы - выявить наличиекорреляцион- ных связей между вариациями атмосферного давления и микросейсмическим фоном на станциях, расположенных на Воронежском кристаллическом массиве. Определить возможность практического применения этих данных, а также возможность их обработки и анализа в реальном времени.

\section{1. МАТЕРИАЛЫ И МЕТОДЫ}

\section{1. Спектральные методы обработки сейсмической информации. Метод осредненного спектра}

В качестве исходных материалов для проведения исследования использовались данные по микросейсмическому фону за срок с 01.09.2017 по 30.11.17, полученные на станциях Новохоперск (VRH) и Сторожевое (VSR). Станции оборудованы сейсмоприемниками CM3-OC. Сейсмоприемники предназначены для преобразования колебаний земной поверхности, вызываемых сейсмическими волнами, в электрический сигнал. «СМ3-OC» является сейсмическим прибором маятникового типа, снабженным магнитоэлектрическим преобразователем. Конструкция корпуса позволяет использовать сейсмоприемник для регистрации как вертикальных составляющих колебательного процесса, так и горизонтальных. Полученные данные были обработаны в программном комплексе WSG [11] и осреднены за трехчасовые промежутки в целях достижения синхронности с отсчетами атмосферного давления на ближайших метеорологических станциях. Значения атмосферного давления на рассматриваемых сейсмических станциях были получены путем интерполяции (аппроксимации) по обратному средневзвешенному расстоянию отсчетов соседних станций. Затем, были рассчитаны корреляционные связи между амплитудой микросейсмического фона в диапазоне частот до 20 Гц с шагом 0,03 Гц и изменением атмосферного давления на рассматриваемых станциях.

В данной статье производится сравнение двух методов (метода осредненного спектра и метода минимальных амплитуд) применитель- 
но к достижению указанной ранее цели. В основе большинства методов применения микросейсмического шума для решения широкого спектра геофизических задач используются его амплитудно-частотные характеристики.

Метод осредненного спектра наиболее широко применим при решении задач анализа микросейсмического фона. Он основан на осреднении гармоник микросейсмического шума, полученных при помощи преобразования Фурье (1) с наложением весового окна (к примеру, Хемминга) (2).

$$
\begin{gathered}
X(k)=\sum_{n=0}^{N-1} e^{-i \frac{2 \pi}{T} k n} x(n)= \\
=\left[\cos \left(\frac{2 \pi}{T} k n\right)-\sin \left(\frac{2 \pi}{T} k n\right)\right] x(n) . \\
w(n)=0.54-0.46 \cos \left(\frac{2 \pi n}{N-1}\right) .
\end{gathered}
$$

Но у этого метода есть ряд недостатков, связанных в первую очередь с селекцией интервалов. Это означает, что из шума убираются сильные помехи, но правило, определяющее исключение помех выбирается непосредственно исследователем, что обуславливает некоторый субъективизм. Кроме того, из-за необходимости постобработки шумов пропадает возможность использования полученных данных в реальном времени [10].

\section{2. Спектральные методы обработки сейсмической информации. Метод минимальных амплитуд}

Амплитудно-частотные характеристики являются, по сути, эмпирическими моделями микросейсмического шума в пунктах измерений. Они рассчитываются из исходных, осложненных помехами сейсмических записей с применением различных математических методов и предварительной селекции, пригодных для расчета интервалов.

В работе [10] разработан метод расчета эмпирической модели микросейсмического фона в пункте наблюдения, который не требует селекции интервалов. Метод основан на экспериментально полученном статистическом свойстве микросейсмического фона для территории Воронежского кристаллического массива (ВКМ): он является непрерывным, однородным и квазистационарным сейсмическим процессом, с постоянным коэффициентом вариации по амплитуде 25 \% для всех гармоник (в рабочем диапазоне частот 0.1-20 Гц).

Путем разбиения непрерывного интервала записи на $k$ равных по длительности окон и вычисления амплитудных спектров $S_{i}(f)$ $(i=1 \ldots k)$ используя дискретное преобразование Фурье, весовое окно Хемминга и сглаживание по 3-м значениям можно по формуле (3) рассчитать частотную характеристику $S(f)$.

$$
S(f)=\left\{\begin{array}{c}
\min \left[S_{1}^{1}, \ldots, S_{1}^{i}, \ldots, S_{1}^{k}\right]\left(f_{1}\right) \\
\ldots \\
\min \left[S_{j}^{1}, \ldots, S_{j}^{i}, \ldots, S_{j}^{k}\right]\left(f_{j}\right) \\
\ldots \\
\min \left[S_{n}^{1}, \ldots, S_{n}^{i}, \ldots, S_{n}^{k}\right]\left(f_{n}\right)
\end{array}\right\}
$$

где $f_{j}$ - частота, а $S_{j}^{i}$ - спектральная амплитуда $i$-го окна для $j$-й частоты.

Свойство дискретного преобразования Фурье позволяет представить амплитудный спектр записи как сумму амплитудных спектров всех действующих сейсмических процессов, среди которых постоянно будет присутствовать только микросейсмический фон. Тогда при достаточной длительности интервала и статистически значимом количестве окон $k$ полученная характеристика $S(f)$ будет включать минимальные амплитуды постоянно присутствующего процесса, т. е. микросейсмического фона.

Сравнение эмпирических моделей МФ для разных пунктов наблюдений на ВКМ показало, что результат расчета модели в каждом пункте платформенной территории достаточно стабилен; модели в разных пунктах имеют значимые различия; а в отдельных диапазонах частот модели совпадают [6]. Таким образом, данный метод можно использовать для выявления корреляционных связей между микросейсмическим фоном и атмосферными параметрами. Расчеты производились с использованием двух представленных выше методов обработки данных. 


\section{3. Программный комплекс WSG}

Для обработки сейсмических данных в ходе исследования использовался программный комплекс WSG. Этот комплекс включает в себя основной программный модуль WSG (Windows Seismic Grafer) и набор вспомогательных сервисных программ-утилит, выполняющихся под управлением операционной системы Windows. Программный комплекс разработан в НПП «Геотех» и Геофизической Службе РАН.

Основные вычислительные процедуры, предназначенные для обработки сейсмических сигналов и получения оценок параметров гипоцентров сейсмических событий как по записям одной станции, так и по группе станций сосредоточены в программном модуле WSG.

Программный модуль WSG работает с данными, предварительно занесенными в виртуальную петлю сейсмических записей, преобразованных во внутренний формат WSG, куда могут быть включены как значительные фрагменты НСИ (до нескольких суток), так и отдельные непродолжительные фрагменты с записью отдельных событий. Для этого в WSG включены программы утилиты-конверторы для различных форматов цифровых сейсмических записей [11].

Тем не менее, существенным недостатком, выявленным в ходе исследования, является отсутствие функции экспорта данных в другом редакторе, что значительно снижает скорость обработки данных.

\section{2. РЕЗУЛЬТАТЫ И ИХ ОБСУЖДЕНИЕ}

На первоначальном этапе исследования был произведен поиск корреляционной связи между атмосферным давлением и сейсмическим шумом по частотам с учетом всех сроков регистрации в течение суток.

На графике, показывающем зависимость коэффициента корреляции от частоты (рис. 1) наглядно видно, что при таком подходе значимой связи между рассматриваемыми величинами практически не наблюдается.

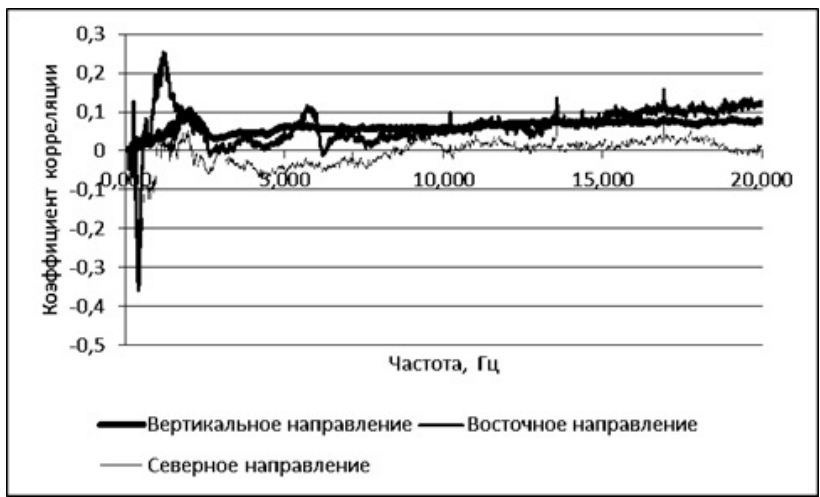

Рис. 1. Зависимость коэффициента корреляиии между рассматриваемыми величинами по всем отсчетам в течение суток.

По убыванию толщины тинии вертикальное, восточное и северное направления регистрации

[Fig. 1. Dependence of the correlation coefficient between the for all counts during the day. Descending line thickness - vertical, eastern and northern directions of registration]

Известно, что антропогенное воздействие (движение автомобилей, функционирование предприятий) вносит помехи в регистрацию сейсмического шума, даже при расположении станции на значительном удалении от источника таких помех. Очевидно, что данный вид помех минимален в течение ночи, поэтомудля того, чтобы минимизировать воздействие антропогенного фактора на следующем этапе исследования учитывались только ночные наблюдения.

В целях сравнения двух описанных ранее методов были рассчитаны корреляционные связи по измененному ряду наблюдений с использованием метода осредненного спектра и метода минимальных амплитуд

На представленном рисунке видно, что в северном направлении коэффициент корреляции, имеющий значение менее $-0,3$ (соответствует умеренной корреляционной связи согласно шкале Чеддока) наблюдается в единичных отсчетах низкочастотной части спектра микросейсмического шума или же не наблюдаются вовсе при расчетах по методу минимальных амплитуд (рис. 2). Тем не менее разность между максимальным и минимальным значением коэффициента корреляции, рассчитанным по методу осредненного спектра практически в два раза превышает этот 
Идентификация корреляиионных связей между метеорологическими параметрами и сейсмическим...

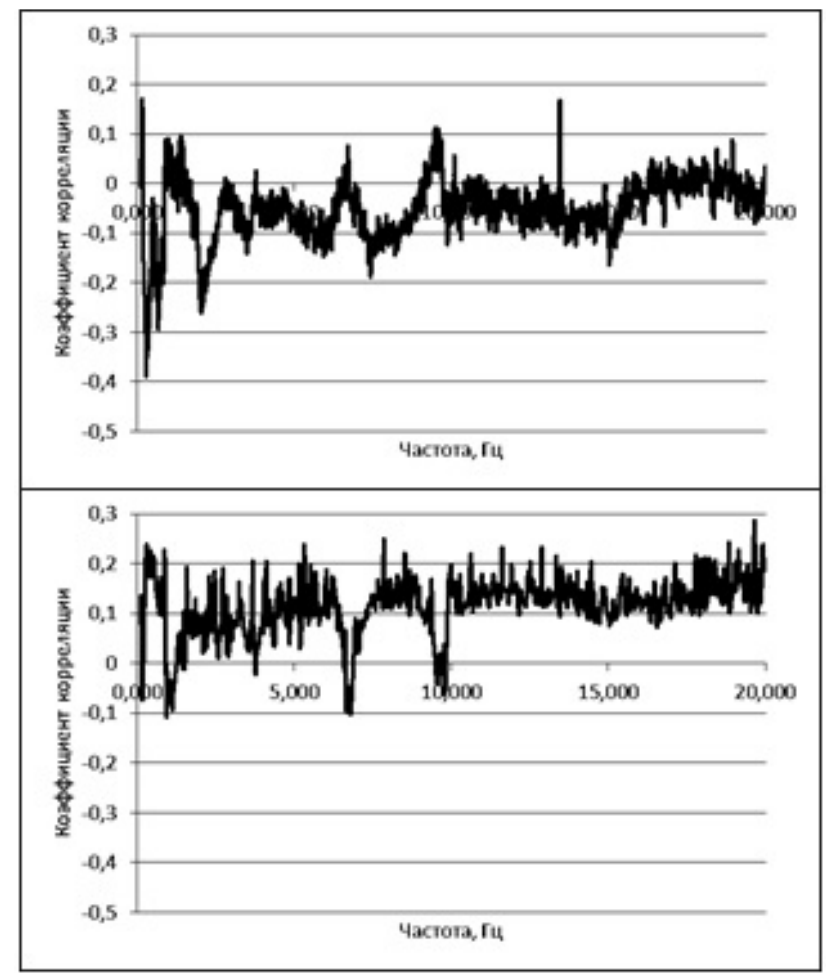

Рис. 2. Зависимость коэббиииента корреляции от частоты (направление на Север). (Сверху - метод минимальных амплитуд, снизу - метод осредненного спектра)

[Fig. 2. Correlation coefficient dependence on frequency (North direction). (From above the method of minimum amplitudes, from below - the method of averaged spectrum)] показатель, полученный при расчетах по методу минимальных амплитуд (порядка 0,6 и 0,3 соответственно).

На рис. 3 представлена зависимость коэффициента корреляции от частоты в вертикальном направлении. Так же, как и на графиках, построенных для северного направления, умеренная корреляционная связь наблюдается на незначительном интервале низкочастотной части спектра. Но разность между максимальным и минимальным значением коэффициента корреляции, рассчитанным по методу осредненного спектра и по методу минимальных амплитуд, возросла и составила 0,5. Предположительно, скачки коэффициента корреляции, наблюдаемые в низкочастотном диапазоне спектра на графиках, полученных по методу осредненного спектра, обусловлены помехами.

Однако в восточном направлении наблюдается иная ситуация. Метод осредненного

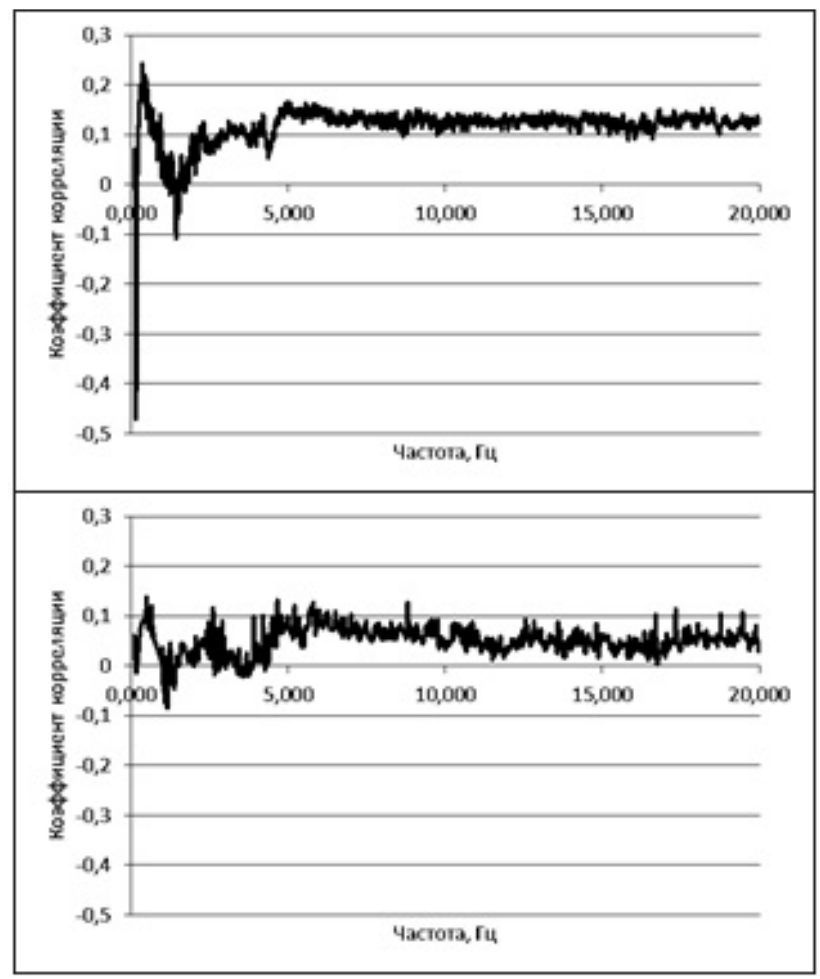

Рис. 3. Зависимость коэфффициента корреляиии от частоты в вертикальном направлении. (Сверху - метод минимальных амплитуд, снизу - метод осредненного спектра) [Fig. 3. Dependence of the correlation coefficient on the frequency in the vertical direction. (From above - the method of minimum amplitudes, from below - the method of averaged spectrum)] спектра дает умеренную корреляционную связь между рассматриваемыми параметрами на более широком интервале в низкочастотной части спектра и приближается к значениям умеренной связи в диапазоне от 6 до 8 Гц. Расчеты по методу минимальных амплитуд показали, что умеренная корреляционная связь наблюдается не только на аналогичном интервале в низкочастотной части спектра, но и на интервале от 6 до 15 Гц. Проанализируем график, представленный на рис. 4 более подробно. Наибольший интервал, коэффициент корреляции на котором менее $-0,3$ заключен между значениями частоты 5,890 до 15,546 Гц. При этом максимальное значение коэффициента равно $-0,49$.

Несмотря на указанные пересечения интервалов сравнительно высокой корреляционной связи, можно прийти к выводу, что метод минимальных амплитуд более стабилен. Как следствие, он достаточно качественно 


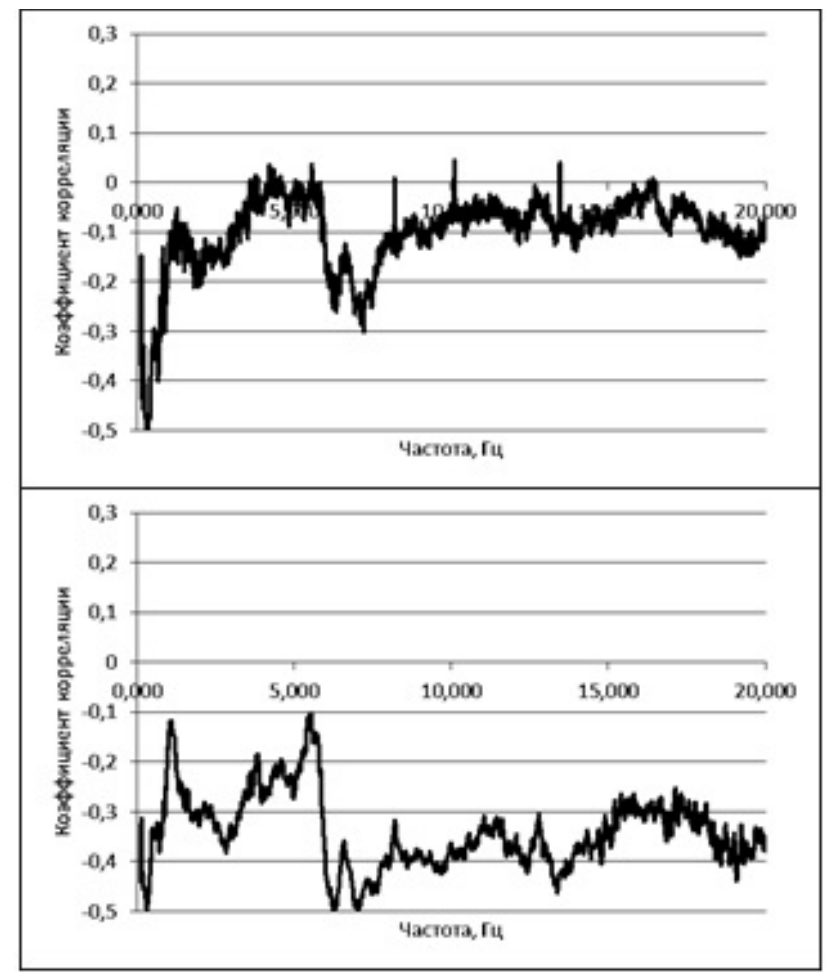

Рис. 4. Зависимость коэфбфициента корреляции от частоты в восточном направлении. (Сверху - метод минимальных амплитуд, снизу - метод осредненного спектра)

[Fig. 4. Dependence of the correlation coefficient on the frequency in the eastern direction. (From above - the method of minimum amplitudes, from below - the method of averaged spectrum)] выделяет искомую реализацию микросейсмического поля на станции - микросейсмический фон. Также, данный метод позволяет выделять фоновую составляющую в реальном времени. Поэтому, для выделения микросейсмического фона на станции Сторожевое (VSR) использовался только метод минимальных амплитуд.

На представленных на рис. 5-7 графиках, показывающих зависимость коэффициента корреляции между вариациями микросейсмического фона и атмосферного давления в вертикальном, северном и восточном направлении для станции Новохоперск отчетливо виден характерный интервал максимального значения коэффициента корреляции между рассматриваемыми величинами (до 3 Гц). Причем в северном и восточном направлении его экстремум приближается к значению 0,7 , являющемуся граничным значением высокой корреляционной связи по шкале Чеддока. Но

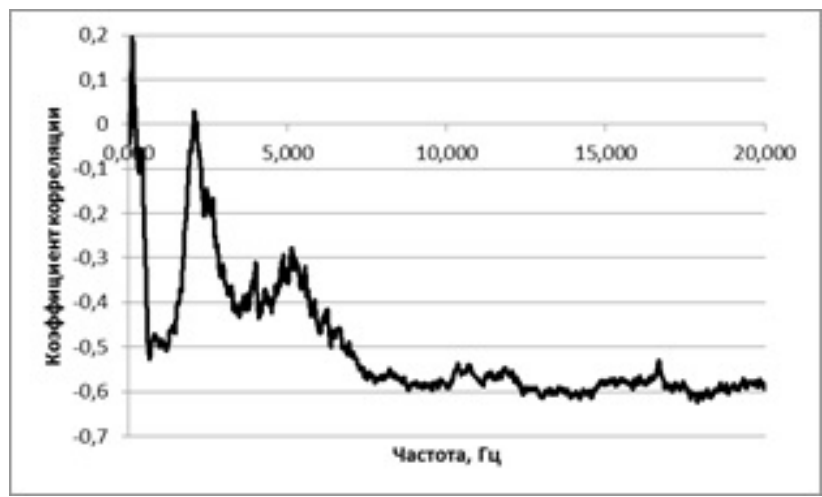

Рис. 5. Зависимость коэффициента корреляици между вариациями микросейсмического фона и атмосферного давления в вертикальном направлении.

[Fig. 5. Dependence of the correlation coefficient between variations of the microseismic background and atmospheric pressure in the vertical direction]

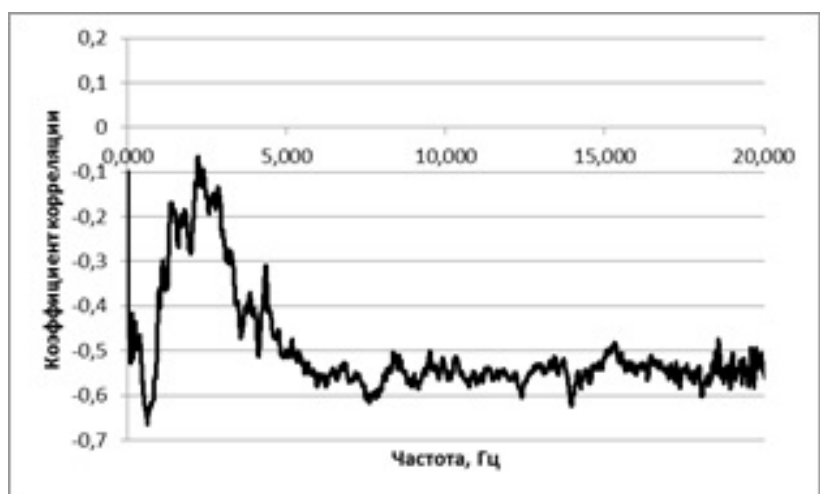

Рис. 6. Зависимость коэффичиента корреляиии между вариациями микросейсмического фона и атмосферного давления в северном направлении.

[Fig. 6. Dependence of the correlation coefficient between variations of the microseismic background and atmospheric pressure in the northern direction]

в отличие от станции Сторожевое, следующий интервал явно выраженной корреляционной связи начинается не от 6 Гц, а от 4 Гц и прослеживается на всем оставшемся исследуемом диапазоне частот до 20 Гц.

Проанализировав полученные результаты можно утверждать, что наличие слабой корреляционной связи между микросейсмическим фоном и вариациями атмосферного давления присутствует на обеих рассматриваемых станциях, при этом в отдельных диапазонах частот (до 3 Гц и более 4-6 Гц) выражена умеренная корреляционная связь. 
Идентификация корреляциионных связей между метеорологическими параметрами и сейсмическим...

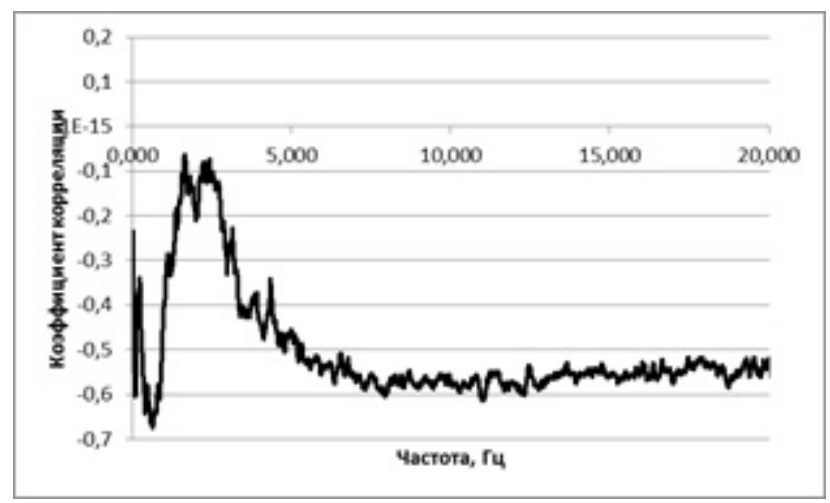

Рис. 7. Зависимость коэфбициента корреляции между вариациями микросейсмического фона и атмосферного давления в восточном направлении

[Fig. 7. Dependence of the correlation coefficient between variations of the microseismic background and atmospheric pressure in the eastern direction]

Также стоит отметить, что различия в тесноте корреляционной связи наблюдаются не только между станциями, находящимися на значительном удалении друг от друга, но и между станциями, принадлежащими одному кристаллическому массиву.

\section{ЗАКЛЮЧЕНИЕ}

Таким образом, в представленной работе было выявлено наличие умеренной и заметной корреляционной связи между характеристиками микросейсмического фона и вариациями атмосферного давления над сейсмическими станциями Новохоперск и Сторожевое. Установлено, что для продолжения исследований на станциях, расположенных на Воронежском Кристаллическом Массиве наиболее применим метод минимальных амплитуд. Было отмечено различие в тесноте этих связей между станциями, принадлежащими одному кристаллическому массиву. Это говорит о том, что для дальнейшего использования полученных результатов необходимо построить корреляционное поле для всего Воронежского кристаллического массива по данным всех расположенных на нем сейсмических станций. Полученные результаты могут лечь в основу нового методического подхода к прогнозу погоды в условиях ограниченной информации.

\section{КОНФЛИКТ ИНТЕРЕСОВ}

Авторы декларируют отсутствие явных и потенциальных конфликтов интересов, связанных с публикацией настоящей статьи.

\section{СПИСОК ЛИТЕРАТУРЫ}

1. Warpinski, N. Microseismic Monitoring: Inside and Out / Warpinski N. // Journal of Petroleum Technology. - November 2009 - Vol. 61, issue 11 .

2. Gal, S. Short Timescale Analysis of Microseisms and Application to Array Calibration / Gal S., Ellingsen P. // Journal of Geophysical Research: Solid Earth. - 2019.

3. Retailleau. Toward High-Resolution Period-Dependent Seismic Monitoring of Tropical Cyclones / Retailleau, Lise\&Gualtieri, Lucia // Geophysical Research Letters. - 2019 - Vol. 46, issue 10

4. van der Baan. Microseismic Monitoring Developments in Hydraulic Fracture Stimulation. / van der Baan, Mirko\& Eaton, David \&Dusseault, Maurice. Geophysical Research Letters. - 2017 - Vol. 25, issue 5.

5. Адушкин, В. В. Влияние барических возмущений атмосферы на микросейсмические процессы в земной коре / В. В. Адушкин, Д. Н. Локтев, А. А. Спивак // Институт динамики геосфер РАН. 2008. - № 6 - С. 77-85.

6. Сафронич, И. Н. Современные методы обработки и интерпретации сейсмологических данных / И. Н. Сафронич, С. А. Красилов, И. М. Колесников, С. И. Колесникова // ГС РАН. - 2017. - С. 386-395.

7. Friedrich, A. Ocean-generated microseismic noise located with the Gräfenberg array / A. Friedrich, F. Krüger \& K. Klinge // Journal of Seismology. - 1998 - Vol. 2, issue 47.

8. Монахов, Ф. И. Низкочастотный сейсмический шум Земли / Ф. И. Монахов // Наука. - 1997.

9. Maxwell, S. Microseismic location uncertainty / S. Maxwell // Focus article. - April 2009. - P. 41-46.

10. Сафронич, И. Н. Метод расчета модели микросейсмического фона / И. Н. Сафронич // ФИЦ ЕГС РАН. - 2017. 
11. Сафронич, И. Н. Анализ данных в частотной области с использованием процессора обработки сигналов программного комплекса WSG / И. Н. Сафронич // ФИЦ ЕГС PAH. - 2017.
12. Антонов, Ю. В. Пульсации силы тяжести и сейсмического шума на Евразийском Континенте / Ю. В. Антонов // Вестник ВГУ: Геология. - 2018. - № 4. - С. 71-76.

Семенов Михаил Евгеньевич - д-р физ.-мат. наук, проф., проф. кафедры теоретической гидрометеорологии, Военного учебного-научного центра военно-воздушных сил «военно-воздушная академия» имени профессора им. Н. Е. Жуковского и Ю. А. Гагарина.

E-mail: mkl150@mail.ru

ORCID iD: https://orcid.org/

Мозиков Михаил Борисович - начальник группы Главного гидрометеорологического центра Министерства обороны Российской Федерации.

E-mail: mb.mozikov@gmail.com

ORCID iD: https://orcid.org/

Сафронич Игорь Николаевич - ведущий инженер Федерального исследовательского центра «Единая геофизическая служба Российской академии наук».

E-mail: igor@geophys.vsu.ru

ORCID iD: https://orcid.org/

DOI: https://doi.org/

Received 20.10.2020

Accepted 02.02.2021

ISSN 1995-5499

\title{
IDENTIFICATION OF CORRELATIONS BETWEEN METEOROLOGICAL PARAMETERS AND SEISMIC NOISE
}

\author{
(C) 2020 M. E. Semenov ${ }^{1,3}$, M. B. Mozikov ${ }^{\bowtie 2}$, I. N. Safronich ${ }^{1,4}$ \\ ${ }^{1}$ Military Training and Research Center of the Air Force "Air Force Academy" \\ named after Professor N. E. Zhukovsky and Yu. A. Gagarin \\ 54a, Stary Bolshevikov Street, 394064 Voronezh, Russian Federation \\ ${ }^{2}$ Main Hydrometeorological Center of the Ministry of Defense of the Russian Federation \\ 22/2, Frunzenskaya Embankment, 119146 Moscow, Russian Federation \\ ${ }^{3}$ Voronezh State University \\ 1, Universitetskaya Square, 394018 Voronezh, Russian Federation \\ ${ }^{4}$ Unified Geophysical Service of the Russian Academy of Sciences \\ 189, Lenin Avenue, 249035 Obninsk, Russian Federation
}

\begin{abstract}
Annotation. The paper analyses correlations between the microseismic background and atmospheric pressure variations in the territory of the Voronezh crystalline massif, which is a platform region. The purpose of the study was to develop a theoretical basis for the method of weather forecasting under conditions of limited meteorological information. The method is based on the registration of fluctuations in filtered microseismic noise - microseismic background.

However, removing the interferences present in microseismic noise is problematic. The main problem in studying the microseismic background field in the platform territory is a high level of interference that exceeds the amplitudes of background oscillations by several times. This means that standard methods cannot completely eliminate the influence of interference on the useful

Mozikov Mikhail B.

e-mail: mb.mozikov@gmail.com signal. The use of "good" intervals for calculating the result makes it subjective, thus reducing its re-
\end{abstract}


liability, which limits the applicability of the background microseismic field as a tool for studying platform territories. In our study, the data was processed using the average spectrum method and the minimal amplitude point method. Also, in order to reduce the impact of anthropogenic factor on the microseismic noise, we selected the fluctuations recorded during the night.

In our study, we analysed and compared the methods used and determined the most optimal approach for further analysis of the interaction between the microseismic background and variations in meteorological characteristics. The most optimal is the minimal amplitude point method. The study also revealed moderate and significant (according to the Chaddock scale) correlation between the considered parameters. We also noted that the degree of this correlations at different stations belonging to the same crystalline massif varied. As a rule, the most pronounced correlation between the microseismic background and atmospheric pressure was observed in the range of up to $3 \mathrm{~Hz}$ and in the range from $4-6$ to $18-20 \mathrm{~Hz}$.

Keywords: microseismic background, microseisms, atmospheric pressure, correlations.

\section{CONFLICT OF INTEREST}

The authors declare the absence of obvious and potential conflicts of interest related to the publication of this article.

\section{REFERENCES}

1. Warpinski N. Microseismic Monitoring: Inside and Out // Journal of Petroleum Technology. November 2009. Vol. 61, issue 11.

2. Gal S., Ellingsen P. Short Timescale Analysis of Microseisms and Application to Array Calibration // Journal of Geophysical Research: Solid Earth. 2019.

3. Retailleau Lise \& Gualtieri Lucia. Toward High-Resolution Period-Dependent Seismic Monitoring of Tropical Cyclones // Geophysical Research Letters. 2019. Vol. 46, issue 10.

4. van der Baan Mirko \& Eaton David \& Dusseault Maurice. Microseismic Monitoring Developments in Hydraulic Fracture Stimulation // Geophysical Research Letters. 2017. Vol. 25, issue 5.

5. Adushkin V. V., Loktev D. N., Spivak A. A. Influence of baric disturbances of the atmosphere on microseismic processes in the Earth's crust //
Institute of Geosphere Dynamics of the Russian Academy of Sciences. 2008. No. 6. P. 77-85.

6. Safronich I. N., Krasilov S. A., Kolesnikov I. M., Kolesnikova S. I. Modern methods of processing and interpretation of seismological data // GS RAS. 2017. P. 386-395.

7. Friedrich A., Krüger $F$. \& Klinge $K$. Ocean-generated microseismic noise located with the Gräfenberg array // Journal of Seismology. 1998. Vol. 2, issue 47.

8. Monakhov F. I. Low-frequency seismic noise of the Earth // Science. 1997.

9. Maxwell S. Microseismic location uncertainty // Focus article. April 2009. P. 41-46.

10. Safronich I. N. Method for calculating the microseismic background model // fitz YGS RAS. 2017.

11. Safronich I. N. Data analysis in the frequency domain using the signal processing processor of the WSG software complex // FITZ YGS RAS. 2017.

12. Antonov Yu. V. Pulsations of gravity and seismic noise on the Eurasian Continent // Vestnik VSU: Geologiya. 2018. No. 4. P. 71-76.

Semenov Mikhail E. - DSc in Physics and Mathematics, Professor, Department of Theoretical Hydrometeorology, N.E. Zhukovsky and Yu.A. Gagarin Air Force Academy.

Email:mkl150@mail.ru

ORCID iD: https://orcid.org/

Mozikov Mikhail B. - team lead, Main Hydrometeorological Centre of the Ministry of Defence of the Russian Federation. Email: mb.mozikov@gmail.com

ORCID iD: https://orcid.org/

Safronich Igor N. - lead engineer, Federal Research Centre Geophysical Survey of Russia Academy of Sciences. Email: igor@geophys.vsu.ru

ORCID iD: https://orcid.org/ 